\title{
Supercritical Fluid Extraction Enhances Discovery of Secondary Metabolites from Myxobacteria
}

\author{
Chantal D. Bader ${ }^{\dagger}$, Markus Neuber $^{\dagger}$, Fabian Panter $^{\dagger}$, Daniel Krug $^{\dagger}$ and Rolf Müller ${ }^{+*}$ \\ † Department Microbial Natural Products, Helmholtz-Institute for Pharmaceutical Research Saarland (HIPS) \\ Helmholtz Centre for Infection Research (HZI), German Center for Infection Research (DZIF, Partnersite Hannover-Braunschweig) and \\ Department of Pharmacy, Saarland University Campus E8.1 , 66123 Saarbrücken (Germany) \\ Correspondence: rolf.mueller@helmholtz-hips.de
}

KEYWORDS: natural products, myxobacteria, supercritical fluid extraction, secondary metabolomics

\begin{abstract}
Supercritical fluid extraction (SFE) is widely used for the isolation of natural products from plants, but its application in efforts to identify structurally and physicochemically often dissimilar microbial natural products is limited to date. In this study we evaluated the impact of SFE on the extractability of myxobacterial secondary metabolites aiming to improve the prospects of discovering novel natural products. We investigated the influence of different co-solvents on the extraction efficiency of secondary metabolites from three myxobacterial strains as well as the antimicrobial activity profiles of the corresponding extracts. For each
\end{abstract}

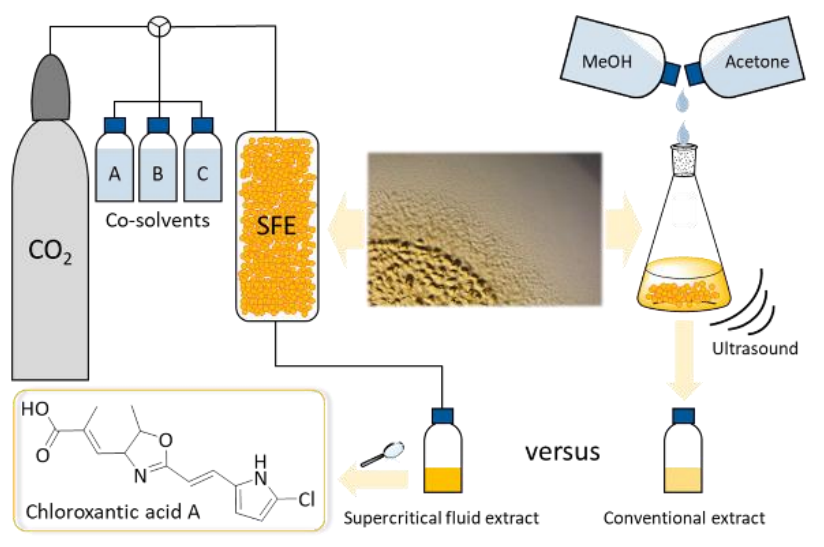
known secondary metabolite we found extraction conditions using SFE leading to superior yields in the extracts compared to conventional solvent extraction. Compounds with a logP higher than 3 showed best extraction efficiency using 20\% EtOAc as a co-solvent, whereas compounds with logP values lower than 3 were better extractable using more polar co-solvents like $\mathrm{MeOH}$. Extracts generated with SFE showed increased antimicrobial activities including the presence of activities not explained by known myxobacterial secondary metabolites, highlighting the advantage of SFE for bioactivity-guided isolation. Moreover, non-targeted metabolomics analysis revealed a group of chlorinated metabolites produced by the well-studied model myxobacterium Myxococcus xanthus DK1622 which were not accessible previously due to their low concentration in conventional extracts. The enriched SF extracts were used for isolation and subsequent structure elucidation of chloroxanthic acid $A$ as founding member of a novel secondary metabolite family. Our findings encourage the increased utilization of SFE as part of future microbial natural products screening workflows

\section{INTRODUCTION}

Over the years, supercritical fluid extraction (SFE) has contributed to efficient extraction processes of plant based natural products for pharmaceutical and food applications. ${ }^{1,2}$ SFE is often characterized as a "green technology" as its benefits include comparably low energy and liquid solvent consumption, relying mainly on supercritical carbon dioxide $\left(\mathrm{CO}_{2}\right)$ as extraction solvent. ${ }^{3}$ Furthermore it profits from the unique intrinsic properties of a supercritical fluid, showing liquid-like solvation because of its liquid-like density, combined with increased mass transfer between the extracted material and the supercritical fluid because of its gas-like viscosity and diffusivity. ${ }^{4}$ These 
unique properties allowed extraction and isolation of a diverse set of natural products from plants, such as flavonoids and phenolic acids from Medicago sativa L. (Alfalfa) ${ }^{5}$ or piperine from Piper nigrum L.. ${ }^{6}$ In microbial natural product research however, the application of SFE appears limited to the isolation of natural products from cyanobacteria. The structural diversity among cyanobacterial metabolites isolated with SFE is high, comprising the epoxide containing polycyclic natural product chaetoglobosin, the xanthone sydowinin B, the nitrophenyl containing mycolutein and luteoreticuls, the glycosylated macrolactam elaiophylin and the polyunsaturated fatty acid $\gamma$-linoleic acid. ${ }^{7,8}$ All of these compounds extractable with dichloromethane in a conventional solvent extraction approach can be enriched with alcohol-modified supercritical $\mathrm{CO}_{2}$ extraction. ${ }^{8}$

Some of the metabolites successfully isolated with SFE from plants or cyanobacteria are not only characterized by their unique chemical properties but exhibit potent biological activities such as the cytotoxic chaetoglobosins., ${ }^{9,10}$ The comparison of chemical profiles and biological activities of SF extracts to conventional solvent extracts has also been carried out successfully for various plants like Satureja montana or Sideritis scardica Griseb.. ${ }^{11,12}$ Compared to soxhlet and hydrodistillation extracts of Satureja montana, SF extracts of this traditional medicine plant show stronger activities against Bacillus cereus and B. subtilis as well as unique activities against Salmonella enteritidis and Colletotrichum coffeanum. ${ }^{11}$ These results highlight the potential impact of SFE on natural products research, as SFE was not only able to generate extracts with improved biological activities, but also enabled generation of extracts showing previously undetected biological activities.

In studies by Silva et al., only supercritical $\mathrm{CO}_{2}$ without the addition of co-solvent was used, which limits the supercritical fluid extraction exclusively to highly non-polar metabolites, as $\mathrm{CO}_{2}$ itself is highly hydrophobic. ${ }^{13}$ Esquivel-Hernández et al. have shown for the cyanobacterium Arthrospira platensis that from all variable extraction parameters like pressure, temperature, co-solvent and the introduction of static, dispersant and dynamic states during the extraction process, the choice of co-solvent has the highest impact on the extraction efficiency. ${ }^{14}$ Pressure and temperature have to be chosen appropriately to exceed the supercritical point of $\mathrm{CO}_{2}$ at $304.12 \mathrm{~K}$ and 73.7 bar. ${ }^{14,15}$ Subsequently, they have to be kept high enough, to preserve the $\mathrm{CO}_{2}$ in supercritical state. For choosing temperature and pressure, it has to be taken into account that increasing percentage of co-solvent increases pressure and temperature required to reach the supercritical point of the mixture. ${ }^{16}$ The exact supercritical point of the respective mixture herein is strongly dependent on the chosen co-solvent. The most widely used co-solvents in SFE are alcohols such as ethanol (EtOH) and methanol (MeOH). ${ }^{3}$

For conventional solvent extraction, the choice of extraction solvent is also the most varied parameter. The extraction process can be enhanced by exposing the extraction mixture to microwaves, ultrasound or heat. Microwaveassisted extraction or heat supported extractions like soxhlet extraction, are not applicable for metabolites suffering from poor thermal stability, given for a broad range of natural products. ${ }^{17}$ SFE in comparison offers the possibility to keep the temperature relatively low due to the additional pressure applied, without loss in extraction efficiency. ${ }^{16}$ On the other hand, conventional solvent extraction methods have one main advantage: they come without expensive instrumentation.

Most microbial natural product isolation protocols rely on maceration as conventional solvent extraction method. ${ }^{18-20}$ To access a preferably diverse set of microbial natural products, Ito et al. exemplarily used a mixture of dichloromethane:methanol:water (64:36:8). ${ }^{18}$ Novel secondary metabolites from myxobacteria isolated in the past years have almost exclusively been extracted with classical solvent extraction methods, using acetone as most commonly used solvent. Besides acetone, only $\mathrm{MeOH}$ and ethyl acetate (EtOAc) were used. ${ }^{21-26}$

Despite its many advantages over solvent extraction, SFE is underrepresented in microbial natural product research and has never been described for the extraction of myxobacterial cells. Most publications dealing with SFE furthermore only report its utilization for previously described natural products, like caffeine or capsaicin. ${ }^{3}$ As a representative example for natural product extraction from microbial secondary metabolism we therefore set out to investigate the metabolome of three different myxobacterial strains using SFE in comparison to conventional 
ultrasound-assisted extraction (UAE) with acetone and $\mathrm{MeOH}$. To study the influence of different co-solvents on the extraction of known myxobacterial metabolites, we performed targeted metabolomics-based examination of the SF extracts. Furthermore, all extracts were compared with regards to detection of antimicrobial activities. As a proof of concept, a novel secondary metabolite only detectable in trace amounts in UA extracts of the wellstudied model organism Myxococcus xanthus DK1622 was isolated and structurally elucidated.

\section{MATERIAL AND METHODS}

All cultivation conditions, analytical methods and statistical data treatment procedures are described in detail in the Supporting Information.

Cultivation and Sample Preparation. Three myxobacterial strains (M. xanthus DK1622, Sorangium sp. MSr11367 and Sandaracinus sp. MSr10575) were grown in 2 liter shaking flasks containing $400 \mathrm{~mL}$ of cultivation medium for 10 days at $30{ }^{\circ} \mathrm{C}$ and $180 \mathrm{rpm}$. An autoclaved aqueous suspension of Amberlite XAD-16 resin was added to all production media to a volumetric concentration of $2 \%[\mathrm{v} / \mathrm{v}]$. The cell pellets and resin were lyophilized prior to further processing. The combined dry pellet containing cells and resin was then pulverized using an electric coffee grinder and split into two equal batches. One batch was extracted by UAE whilst the other batch was extracted with SFE. Blank extracts were generated by extracting the medium with resin.

Extraction procedure. SFE was carried out on a Waters ${ }^{\circ} \mathrm{MV}-10$ ASFE $^{\circ}$ system equipped with $10 \mathrm{~mL}$ analytical extraction cartridges. The pulverized pellets were densely packed into the cartridges and topped up with glass beads to avoid pressure drops due to inconsistencies in the packing. The extraction process was carried out at 200 bar and $40{ }^{\circ} \mathrm{C}$ using a total flow rate of $10 \mathrm{~mL} / \mathrm{min}$. The extraction was done in five steps, each one performed with an initial dynamic extraction step for 5 minutes followed by a static extraction step for 5 minutes and a second dynamic extraction step for another 5 minutes. The pellets were first extracted with $100 \% \mathrm{CO}_{2}$. Afterwards, the pellet was extracted with $20 \%$ EtOAc as co-solvent, followed by $20 \%$ isopropanol ('PrOH), $20 \% \mathrm{MeOH}$ and as a final step the pellet was flushed with $50 \% \mathrm{MeOH}$. The $\mathrm{MeOH}$ make-up flow was set to a total organic solvent flow of 3 $\mathrm{mL} / \mathrm{min}$. All steps were collected and treated separately. The solvents were evaporated under reduced pressure and the residues were dissolved in $4 \mathrm{~mL} \mathrm{MeOH}$ each. UAE consisted of two subsequent extraction steps. Firstly, $100 \mathrm{~mL} \mathrm{MeOH}$ was added and the pellet sonicated for $1 \mathrm{~h}$. After removal of the $\mathrm{MeOH}, 100 \mathrm{~mL}$ acetone was added, and the pellet was sonicated for another $1 \mathrm{~h}$. The two extracts were combined, and the solvent was removed under reduced pressure. The residues were dissolved in $4 \mathrm{~mL} \mathrm{MeOH}$ each. All extracts were diluted 1:100 prior to UHPLC-MS measurements.

Isolation procedure. Chloroxanthic acid A was isolated by semipreparative HPLC following a fractionation of the crude extract by liquid-liquid partitioning. Purification was performed on a Dionex Ultimate 3000 SDLC low pressure gradient system equipped with a Phenomonex Luna ${ }^{\circledR} 5 \mu \mathrm{m}$ C18(2) $100 \AA$ A LC Column $(250 \times 10 \mathrm{~mm})$ thermostated at $45^{\circ} \mathrm{C}$. Separation was achieved using a linear gradient from $95 \%$ (A) dd $\mathrm{H}_{2} \mathrm{O}$ with $0.1 \%$ formic acid to $70 \%$ (B) acetonitrile with $0.1 \%$ formic acid over 29 minutes. The compound was detected by UV absorption at 210 and $280 \mathrm{~nm}$.

Analytical analysis of crude extracts and pure compound. UHPLC-qTOF measurements were performed in duplicates as technical replicates on a Dionex Ultimate $3000 \mathrm{SL}$ system coupled to a Bruker maXis 4G UHRqTOF. The mobile phase consisted of $(A) d_{d H_{2}} \mathrm{O}$ with $0.1 \%$ formic acid and (B) acetonitrile with $0.1 \%$ formic acid. For separation a linear gradient from 5-95\% B in A on a Waters Acquity BEH C18 column (100 x $2.1 \mathrm{~mm}, 1.7 \mu \mathrm{m} \mathrm{d}$ ) was used. Flow rate was set to $0.6 \mathrm{~mL} / \mathrm{min}$ and the column heated to $45^{\circ} \mathrm{C}$. The LC flow was split to $75 \mu \mathrm{L} / \mathrm{min}$ before entering the mass spectrometer, which was externally calibrated to a mass accuracy of $<1 \mathrm{ppm}$. External calibration was achieved with sodium formate clusters. Mass spectra were acquired in centroid mode ranging from 150-2500 $\mathrm{m} / \mathrm{z}$ at a $2 \mathrm{~Hz}$ scan rate. Capillary voltage was set to $+4000 \mathrm{~V}$ for measurements in positive ionization mode. Dry gas was set to a flow rate of $5 \mathrm{~L} / \mathrm{min}$ at $200^{\circ} \mathrm{C}$. 
NMR spectra were recorded on a Bruker Ascend 700 spectrometer equipped with a $5 \mathrm{~mm}$ TXI cryoprobe $\left({ }^{1} \mathrm{H}\right.$ at $700 \mathrm{MHz},{ }^{13} \mathrm{C}$ at $175 \mathrm{MHz}$ ). All observed chemical shift values $(\delta)$ are given in in ppm and coupling constant values $(J)$ in $\mathrm{Hz}$. Standard pulse programs were used for HMBC, HSQC and gCOSY experiments. HMBC experiments were optimized for ${ }^{2,3} J_{C-H}=6 \mathrm{~Hz}$. The spectra were recorded in methanol- $d_{4}$ and chemical shifts of the solvent signals at $\delta_{\mathrm{H}} 3.31 \mathrm{ppm}$ and $\delta_{\mathrm{C}} 49.2 \mathrm{ppm}$ were used as reference signals for spectra calibration. To increase sensitivity, the measurements were conducted in a 5 mm Shigemi tube (Shigemi Inc., Allison Park, PA 15101, USA).

Statistical Analysis and Annotations. Statistical interpretation of the LC-MS data for targeted and non-targeted metabolomics analysis was carried out with MetaboScape 4.0 (Bruker). The minimal intensity threshold for feature detection by the built-in T-Rex 3D algorithm was set to $1.5 \times 10^{4}$ and the maximum charge was set to three. The minimal group size for creating batch features to five. Known myxobacterial secondary metabolites were annotated using our in-house database containing myxobacterial secondary metabolites (Myxobase). Overlap of the extraction methods as well as features uniquely found in one analysis were calculated via t-Test analysis in MetaboScape 4.0.

Determination of antimicrobial activities. For determination of the extracts' antimicrobial activities, all three biological replicates were combined and $20 \mu \mathrm{L}$ of the saturated methanolic extract was pipetted to $150 \mu \mathrm{L}$ microbial test culture and serially diluted in 96 well plates. The microbial test panel consisted of Staphylococcus aureus Newman, Escherichia coli DSM111, E. coli TolC, Bacillus subtilits, Micrococcus luteus, Pseudomonas aeruginosa PA14, Candida albicans, Pichia anomala, Mucor hiemalis and Mycobacterium smegmatis Mc2155.

\section{RESULTS AND DISCUSSION}

We generated a total number of six different extracts per strain. In case of the SF extraction, the pellet was extracted sequentially with pure $\mathrm{CO}_{2}$, followed by the addition of $20 \% \mathrm{EtOAc}$, ${ }^{\mathrm{P} P O H}$ and $\mathrm{MeOH}$ as co-solvents. The co-solvents were used in ascending polarity. As the last step $50 \% \mathrm{MeOH}$ was used in addition to the $20 \% \mathrm{MeOH}$ step. When SF extracts are referred to in the following, this is indicated by the percentage and type of cosolvent used. Conventional extraction is referred to as ultrasound assisted extraction (UAE).

Influence of the co-solvent on the extraction of myxobacterial secondary metabolites. All extracts were investigated in a targeted metabolomics workflow and known myxobacterial secondary metabolites were annotated from our in-house data base (Myxobase). As Sandaracinus MSr10575 and Sorangium strain MSr11367 are new myxobacterial strains that were recently isolated in our working group, a lower number of metabolites is known compared to the well-studied model myxobacterium M. xanthus DK1622. ${ }^{27,28}$ In M. xanthus DK1622 extracts, five different natural product families, namely the cittilins, myxovirescins, DKxanthenes, myxalamides and myxochromids, are detected. Representatives of these natural product families are shown in Figure 1 (A-E). The remaining derivatives can be found in in the SI. Myxochelins and myxoprincomides, two additional natural product families known from this strain, are not produced under the chosen cultivation conditions. Besides those secondary metabolites, the strain is found to produce riboflavin. As there is a controversial debate if riboflavin should be taken as primary or secondary metabolite (sometimes classified as pseudo-secondary metabolite), the production and extraction is just mentioned for completeness but not discussed in more detail here. ${ }^{29}$ Both described cittilin derivatives ( $A$ and $B$ ) could be detected in the extracts, with the highest intensity found in the $50 \% \mathrm{MeOH}$ extracts. ${ }^{30,31}$ Cittilins are also extractable with $20 \% \mathrm{MeOH}$ as well as UAE and cittilin A can also be detected in lower intensities in the $\mathrm{PrOH}$ extract. The family of myxovirescins contains more than 12 members, whereof six different derivatives ( $\mathrm{A}-\mathrm{C}$ and $\mathrm{G}-\mathrm{I}$ ) are detectable in our analysis. ${ }^{32}$ The highest intensities of all myxovirescin derivatives are found in the EtOAc extracts. Contrary to the cittilins that are not extractable with pure $\mathrm{CO}_{2}$, the myxovirescins are also extractable without the addition of any co-solvent in SFE. They are also present in low amounts in the $20 \%$ and $50 \% \mathrm{MeOH}$ extracts. Due to the serial design of the experiment, this indicates that the myxovirescins could be extracted using $\mathrm{MeOH}$ as a co-solvent, but the compounds were already exhaustively extracted during the EtOAc step. Another abundant and derivative-rich family of secondary metabolites are the DKxanthenes (DKx). ${ }^{33}$ 

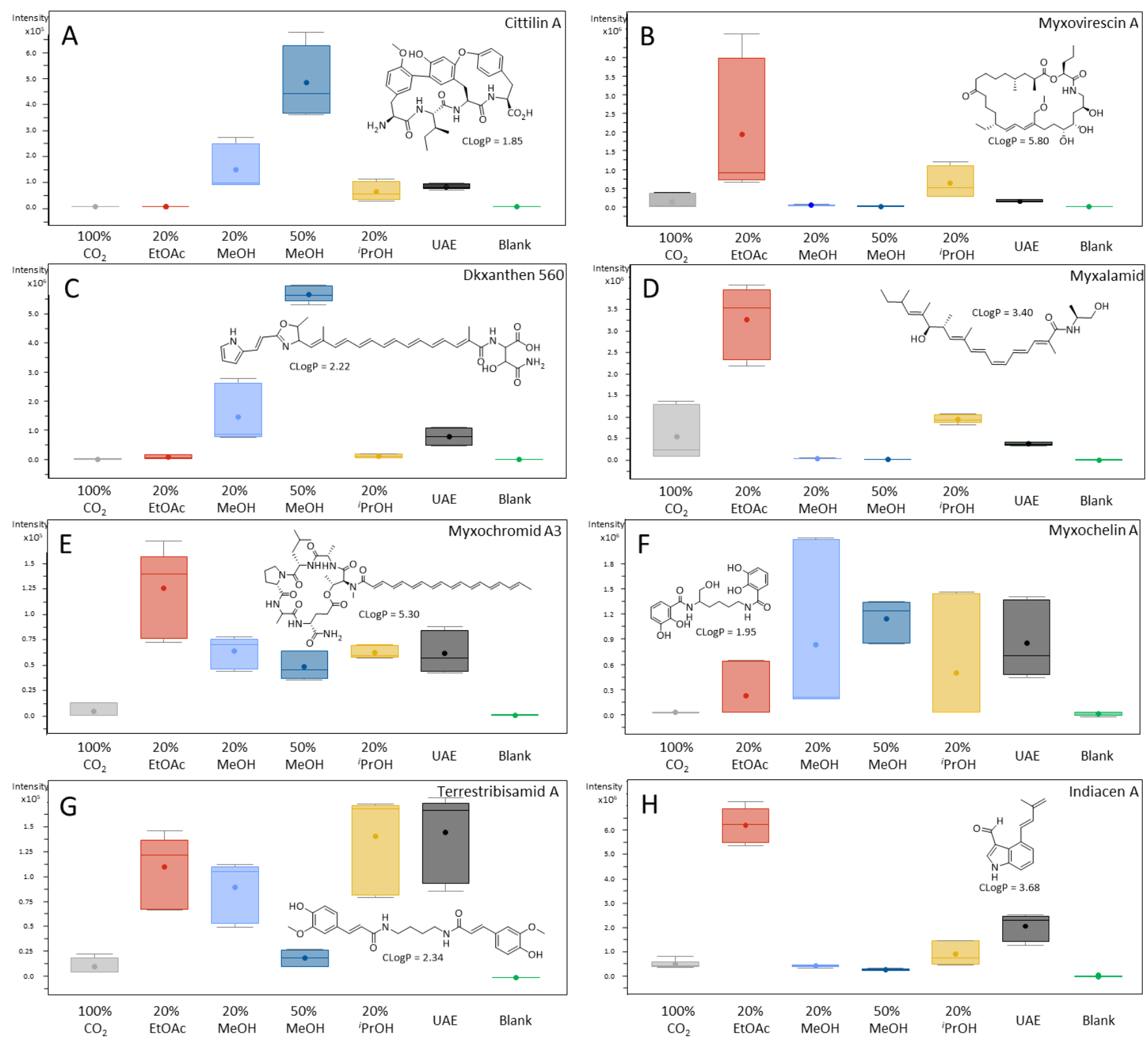

Figure 1 Influence of the co-solvent on the supercritical fluid extraction of secondary metabolites from M. xanthus DK1622 (A-E) and MSr10575 (F-H). Detected intensities are shown as box plots. From left to right: gray $100 \% \mathrm{CO}_{2}$, red $20 \%$ EtOAc, light blue $20 \% \mathrm{MeOH}$, dark blue $50 \% \mathrm{MeOH}$, yellow $20 \%$ i PrOH, black UAE and green combined blank (SFE+UAE).

In our analysis, we detect seven DKx derivatives, DKx 520, 526, 534, 544, 548, 560 and 574. Except for DKx 526 all derivatives show the highest intensities in the $50 \% \mathrm{MeOH}$ extracts. DKx 526 on the other hand shows highest intensities in the EtOAc extract. All described myxalamide derivatives (A-D and K) are detectable in our extraction analysis, with highest intensities in the EtOAc extracts. The ${ }^{i} \mathrm{PrOH}$ extracts show higher intensities than the UAE as well. ${ }^{34,35}$ From the myxochromide compound family, only one derivative (A3) is observed. ${ }^{36}$ Here, we find highest intensities in the EtOAc extracts. Myxochromide $\mathrm{A} 3$ is also found in the ${ }^{\mathrm{P}} \mathrm{PrOH}$ and both of the $\mathrm{MeOH}$ extracts. In those extracts the detected intensities of the secondary metabolite are on a similar level as in the UAE, indicating that only SF extraction using EtOAc is superior in extraction efficiency compared to UAE in this case. In the Sandaracinus MSr10575 extracts, three known myxobacterial secondary metabolite families are detectable (Figure 1 $\mathrm{F}-\mathrm{H})$. From the two natural myxochelins $\mathrm{A}$ and $\mathrm{B}$ only myxochelin $\mathrm{A}$ is observed in the extracts. Highest intensities are found in the $20 \% \mathrm{MeOH}$ extracts, but the compound is present in all other extracts except the $100 \% \mathrm{CO}_{2}$ extract. Terrestribisamid $A$ is the only known secondary metabolite in our extracts showing the highest intensity in one of the UA extracts. ${ }^{37}$ Nevertheless, this compound is also extractable with SFE, whereby ${ }^{i} \mathrm{PrOH}$ shows the 
best extraction efficiency. Indiacen A and B, two secondary metabolites originally isolated from the myxobacterial strain Sandaracinus amylolyticus NOSO-4T also belonging to the family of Sandaracineae are also found in the extracts of MSr10575. ${ }^{38}$ The Indiacens show highest intensities in the EtOAc extracts. As those prenylated indole aldehydes with activity against Gram-positive and Gram-negative bacteria as well as the fungus Mucor hiemalis are the only reported secondary metabolites from another Sandaracinus strain to date, we wanted to validate our initial hit based on exact mass and retention time and isolated both derivatives. Crystallization followed by X-ray crystallography of the isolated compounds confirms that MSr10575 is indeed able to produce Indiacens (see SI).

All known secondary metabolites described above are extractable with UAE as a conventional extraction method and SFE. Concerning the different co-solvents investigated, EtOAc and $\mathrm{MeOH}$ are found to be the most efficient co-solvents for extraction. $\mathrm{MeOH}$-based SF extracts show specificity for more polar myxobacterial secondary metabolites, while EtOAc as a co-solvent leads to increased extraction of more nonpolar secondary metabolites. Furthermore, extraction with $50 \% \mathrm{MeOH}$ as co-solvent seems to be superior to the extraction with $20 \% \mathrm{MeOH}$. Due to the relatively high pressure of 200 bar in our experiments, the $\mathrm{CO}_{2} / \mathrm{MeOH}$ mixture remains in the supercritical state even when increasing to an equipotential portion of the co-solvent. This is reflected in the high extraction efficiency for more polar natural products such as the cittilins and the DKxanthenes. ${ }^{39,40}$ Our data suggests that secondary metabolites such as myxovirescin A (CLogP $=5.80)$, myxalamid A (CLogP = 3.40), myxochromid A3 (CLogP $=5.30)$ and indiacen $A(C L o g P=3.68)$ exceeding a $\log P$ of 3 show best extraction using $20 \%$ EtOAc as a co-solvent. Secondary metabolites such as cittilin A (CLogP = 1.85), DKxanthen 560 (CLogP = 2.22), myxochelin A (CLogP = 2.34) and terrestribisamid $A(C \log P=2.34)$ with $\log P$ values below 3 are better extractable using more polar and protic co-solvents such as ${ }^{\mathrm{P}} \mathrm{PrOH}$ and $\mathrm{MeOH}$ (see Figure 1 ).

Antimicrobial activities of the SF and UA extracts. All of the three examined myxobacterial strains show potent activities against at least three test organisms of our antimicrobial test panel (see Figure 2). Extracts of Sandaracinus MSr10575 display the highest and broadest activities, with antimicrobial activities of at least one of the extracts against each of the test organisms. The conventional UA extract shows activities against S. aureus, E. coli, E. coli TolC (representing an efflux deficient E. coli strain), B. subtilis, M. luteus, P. aeruginosa and M. smegmatis. All of these activities are also found in the $20 \% \mathrm{MeOH}$ and $50 \% \mathrm{MeOH}$ SF extracts, indicating that the responsible metabolites are relatively polar. Interestingly, the $100 \% \mathrm{CO}_{2}$ extracts show a selective activity against Gram-positive pathogens as well as $C$. albicans. As those activities do not correlate to the ones observed in other extracts, it is very likely that they are caused by a different family of natural products. Furthermore, we observe activities of the 'PrOH based SF extract against M. luteus, M. smegmatis and C. albicans. The EtOAc extract on the contrary shows a selective activity against $M$. smegmatis. Besides the activities of the ${ }^{i} \mathrm{PrOH}$ extract against $M$. luteus and C. albicans all activities are comparably strong, showing an inhibition down to a dilution of the extract to $0.1 \%$ of the starting concentration. This indicates that the selectivity of antimicrobial activities does not result from the inaccuracy of the assay or the presence of compound yields close to their minimum inhibitory concentration (MIC), but rather the presence of several different antimicrobial natural product classes in the extract. From the known myxobacterial secondary metabolites produced by this strain, only indiacen is known for antimicrobial activity. ${ }^{38}$ Although it was not tested against $M$. smegmatis itself, it exhibits activities against two related mycobacterial species. By far the highest intensities of indiacens are measured in our EtOAc extracts. This finding correlates with the selective inhibition of $M$. smegmatis by the EtOAc extract. Nevertheless, the strong other antimicrobial activities cannot be explained by the presence of already described secondary metabolites alone, making this strain a target for further secondary metabolite extraction campaigns.

The UA extract of $M$. xanthus DK1622 shows activities against $E$. coli TolC, explained by the presence of myxovi-

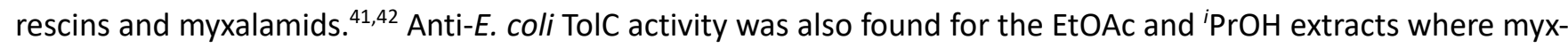
ovirescins and myxalamids are present in higher intensities than in the UAE. Furthermore, those two extracts show 

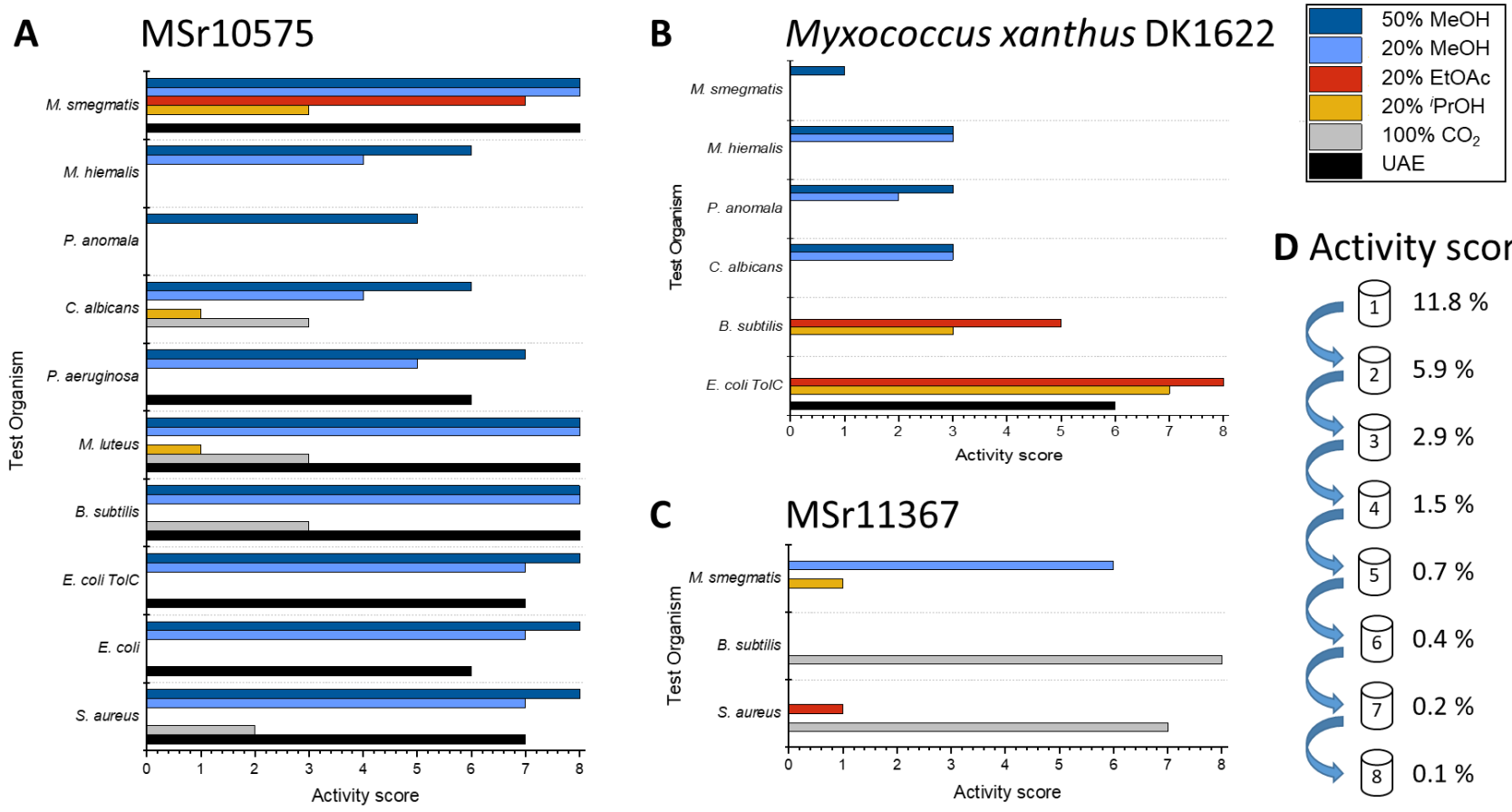

D Activity score

C
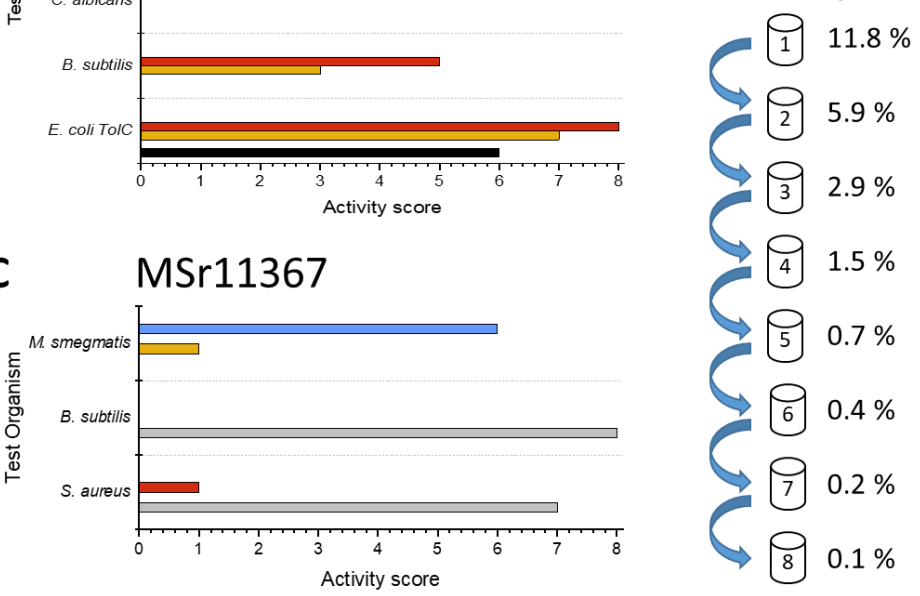

Figure 2 Antimicrobial activity score of myxobacterial SF extracts in comparison to conventional UA extraction (black). A MSr10575, B M. xanthus DK1622, C MSr11367. Extract with $50 \% \mathrm{MeOH}$ as co-solvent is shown in dark blue, $20 \% \mathrm{MeOH}$ in light blue, $20 \% \mathrm{EtOAc}$ in red, $20 \%$ iPrOH in yellow and $100 \% \mathrm{CO}_{2}$ in grey. D Activity score with correlating concentration of the crude extract showing an inhibition of the test organism.

activity against $B$. subtilis, explained by the presence of the same natural product classes. In the UA extract the concentration of those secondary metabolites very likely does not reach the minimal inhibitory concentration, wherefore no activity against $B$. subtilis is detectable. We additionally observe antifungal activities of the $\mathrm{SF} \mathrm{MeOH}$ extracts as well as antimycobacterial activity only observed for the $50 \% \mathrm{MeOH}$ extract. None of these activities can be correlated to known secondary metabolites detectable in the crude extract, highlighting that the potential of secondary metabolite discovery even in well-described strains like M. xanthus DK1622 is far from exhausted.

MSr11367 only shows few activities compared to the other two strains. Contrary to the other myxobacterial strains we observe no antimicrobial activities in the UA extract at all. The $100 \% \mathrm{CO}_{2}$ extract of this strain, however, shows the highest antimicrobial activities when just comparing the $100 \% \mathrm{CO}_{2}$ extracts of the different strains and represents the only case where we can detect an activity selectively in the extract without co-solvent. Furthermore, we observe a strong activity of the $20 \% \mathrm{MeOH}$ extract against $M$. smegmatis besides weak activities of the iPrOH extract against $M$. smegmatis and of the EtOAc extract against $S$. aureus. In all our extracts from MSr11367 we were not able to detect any known myxobacterial natural product, wherefore these activities offer the possibility for bioactivity-guided isolation of novel secondary metabolites.

In summary, for all three myxobacterial strains we were able to enhance the detectability of biological activities using SFE instead of UAE. Furthermore, each of the strains showed additional activities for the SF extracts, not observable for the UA extracts.

Non-targeted metabolomics investigation of the extracts. Besides the targeted metabolomics investigation of the three myxobacterial strains' extracts as well as their biological characterization, we sought to access the total chemical complexity of the different extracts to fully characterize overlaps and differences of the two extraction systems as well as the influence of the different co-solvents. We therefore performed a non-targeted metabolomics analysis and compared features detected in the UAE with those detected in the SF extracts. Furthermore, we compared features detected in the individual SF extracts resulting from the use of different co-solvents. The 
results of this investigation for strain MSr10575 are depicted in Figure 3 as Euler diagrams. Results for the two other strains can be found in the SI.

The overlap of features found with UAE and SFE is very high. $92 \%$ of the total features can be extracted with both methods. Looking at the features uniquely extractable with one of the methods, about $12 \%$ more features are only present in the SF extract than in the UA extract. If it is only a matter of accessing as many features as possible with one method, SFE should therefore be the method of choice. The comparison of the different co-solvents used for the extraction shows that the number of metabolites extractable with only one co-solvent is relatively low. Among the different co-solvents, extraction with $50 \% \mathrm{MeOH}$ yielded the most features. In general, most of the metabolites are detectable in all of the SF extracts (885 features) or in all of the SF extracts except the $\mathrm{CO}_{2}$ extract (909 features). Omitting the first extraction step with $100 \% \mathrm{CO}_{2}$ would only result in a data loss of less than $1 \%$ as all other metabolites are also accessible with the addition of co-solvents and should therefore be preferred if the extraction protocol needs to be shortened.

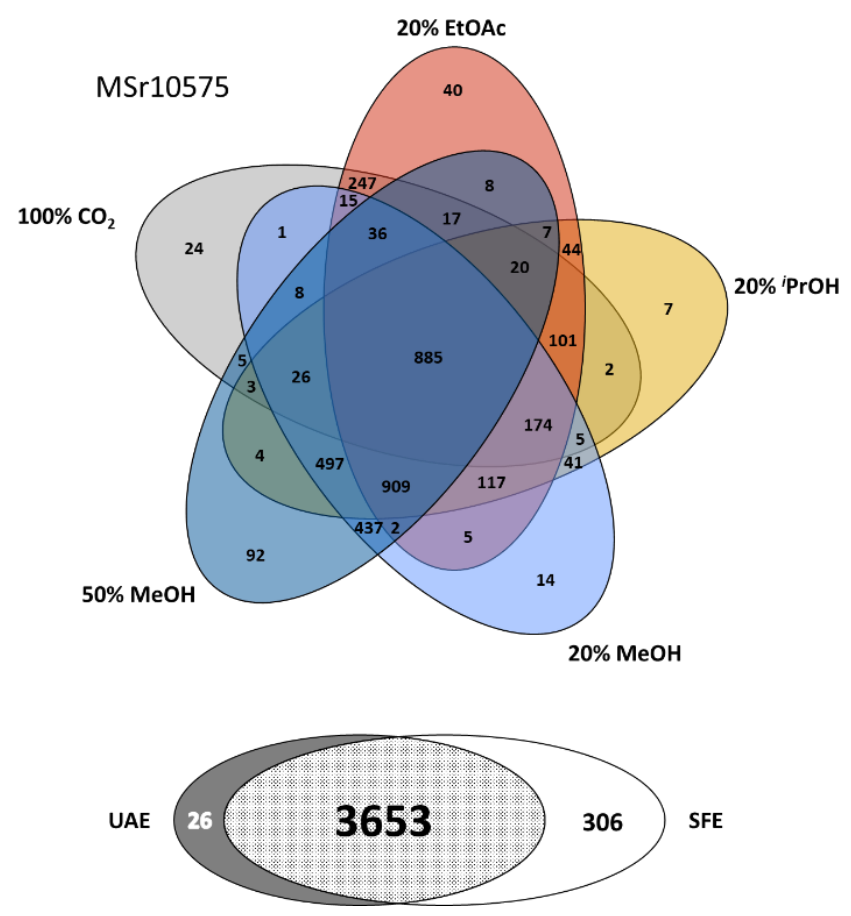

Figure 3 Upper part: Euler diagram showing features found in the SF extracts from MSr10575 sorted by co-solvent. Intersections of the extracts generated using different co-solvents are shown in the same colors as used in Fig. 1 and 2. Lower part: Euler diagram showing features unique for UAE or SFE and features extractable with both methods.

During the non-targeted investigation of the SF extracts of M. xanthus DK1622 a group of metabolites raised our interest. They all show significantly higher intensities in the SF extract using EtOAc as a co-solvent and an isotopic pattern characteristic for chlorination as depicted in Figure 4. Halogenated natural products are well-known to exhibit diverse biological activities, making those features particularly interesting for further investigation. The presence of 30 features with characteristic chlorination-indicating isotope pattern furthermore indicated, that they belong to a relatively large family of secondary metabolites (see $\mathrm{SI}$ ). According to MS analysis all family members incorporate a minimum of two nitrogen and two oxygen atoms. To investigate the chemical structure of those compounds, we isolated the main derivative, later named chloroxanthic acid $A$. As the other compounds are only produced in comparably low amounts, they were not accessible to full structure elucidation by NMR. 


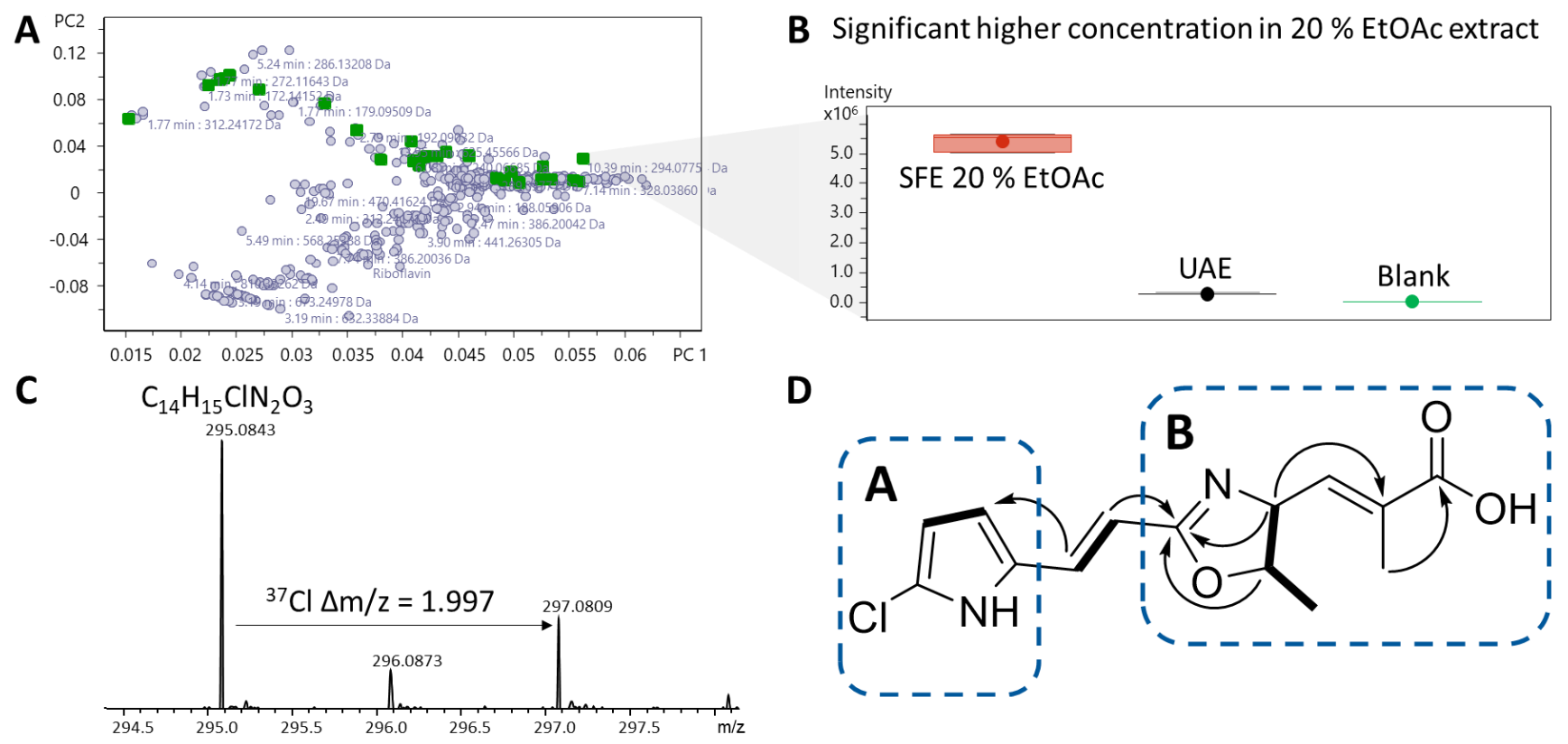

Figure 4 Chlorinated metabolites from M. xanthus DK1622. A PCA plot of the comparison between UAE and SFE 20\% EtOAc extracts. Chlorinated features are highlighted in green. B Box plot for chloroxanthic acid A highlighting the differences in intensities between UAE and SFE EtOAC extract. C Zoom into the isotopic pattern of the $[\mathrm{M}+\mathrm{H}]^{+}$peak of chloroxanthic acid $A$ showing the characteristic mass shift of the ${ }^{37} \mathrm{Cl}$ isotope. D Structure of chloroxanthic acid A. Most relevant COSY correlations are marked bold and most relevant $\mathrm{HMBC}$ correlations are shown as arrows. Substructures $A$ and $B$ are marked in dashed blue boxes.

Structure elucidation of chloroxanthic acid A. HRESIMS of chloroxanthic acid A shows an $[\mathrm{M}+\mathrm{H}]^{+}$peak at $\mathrm{m} / \mathrm{z}$ 295.0848 consistent with the molecular formula $\mathrm{C}_{14} \mathrm{H}_{15} \mathrm{ClN}_{2} \mathrm{O}_{3}\left(\mathrm{~m} / z\right.$ calcd for $\left.[\mathrm{M}+\mathrm{H}]^{+} 295.0844\right)$ containing 8 double-bond equivalents (DBE). The ${ }^{1} \mathrm{H}-\mathrm{NMR}$ spectrum of chloroxanthic acid $\mathrm{A}$ exhibits five signals characteristic for double bond protons at $\delta=7.14(1 \mathrm{H}, \mathrm{d}, J=16.18 \mathrm{~Hz}), \delta=6.38(2 \mathrm{H}, \mathrm{m}), \delta=6.18(1 \mathrm{H}, \mathrm{d}, J=16.09 \mathrm{~Hz}), \delta=6.03(1 \mathrm{H}$, $\mathrm{d}, J=3.66 \mathrm{~Hz})$. Furthermore, two methyl groups at $\delta=1.94(3 \mathrm{H}, \mathrm{d}, J=1.44 \mathrm{~Hz})$ and $\delta=1.43(3 \mathrm{H}, \mathrm{dd}, J=7.00 \mathrm{~Hz})$ and two methine groups at $\delta=4.55(1 \mathrm{H}, \mathrm{dd}, J=9.30, J=7.18 \mathrm{~Hz})$ and $\delta=4.46(1 \mathrm{H}, \mathrm{m})$ are observed. Their coupling constants as well as COSY correlations reveal the two protons at $\delta=7.14$ and $\delta=6.18$ to be part of an aliphatic double bond in $E$-configuration. Their down-field chemical shift suggests participation in a bigger conjugated $\pi$ system. HMBC correlations show their location between two different substructures (substructure $A$ and $B$ ) of the molecule (see Error! Reference source not found.). COSY and $\mathrm{HMBC}$ correlations reveal, that substructure A consists of the two double bond protons at $\delta=6.03$ and $\delta=6.38$ as well as two quaternary carbons at $\delta=129.8$ and $\delta=120.2$. Their characteristic chemical shifts suggest that substructure $A$ is a pyrrole ring with chlorination at the quaternary carbon at $\delta=120.2$ and the quaternary carbon at $\delta=129.8$ is the linker to the aliphatic double bond. The splitting pattern of the two protons of the pyrrole moiety as well as their COSY correlations confirm their allocation next to each other, leaving only two possibilities for substitution of the pyrrole ring. The downfield shifts of both the chlorine bearing carbon at $\delta=120.2$ and the quaternary carbon at $\delta=129.8$ finally reveal chlorination in 5 position of the pyrrole ring. In substructure B the two aliphatic double bond protons only show HMBC correlations to one quaternary carbon at $\delta=166.8$. Its characteristic chemical shift as well as further HMBC correlations to the two methine groups at $\delta=4.55$ and $\delta=4.46$ suggest an oxazoline core structure. COSY correlations reveal a substitution with the methyl group at $\delta=4.46$ in the 5 position. The 4 position of the oxazoline moiety is linked to 2-methylacrylic acid, which are confirmed by COSY and HMBC correlations.

Chloroxanthic acid A shows partial structural similarity to the DKxanthenes that were already described from $M$. xanthus DK1622 before. ${ }^{33}$ The DKxanthenes are produced by a hybrid polyketide synthase - non-ribosomal peptide synthetase (PKS-NRPS) gene cluster. ${ }^{43}$ The two NRP parts of the molecules are linked by a polyketide chain 
consisting of an aliphatic conjugated double bond system in variable chain lengths. Chloroxanthic acid A only comprises the first NRP part of the DKxanthenes, elongated with one methylmalonyl-CoA unit of the PK part. To the best of our knowledge, halogenated DKxanthene derivatives have not been described previously, nor is a halogenase part of the reported biosynthetic gene clusters of those natural products. ${ }^{33,43}$ The isolation and structure elucidation of chloroxanthic acid A highlights the impact of applying deviant analytical chemistry approaches for the discovery of novel natural products from already well-studied bacterial strains. Besides the setup chosen for the detection of secondary metabolites as shown by the discovery of the myxoprincomides by Cortina et al., the extraction method has a tremendous influence on the accessibility of natural products.

\section{CONCLUSION}

SFE in microbial secondary metabolomics is a promising yet underexplored technique for the discovery and extraction of new natural products. In our study we were able to show that for each known metabolite in our three myxobacterial strains, supercritical $\mathrm{CO}_{2}$ extraction efficiency was comparable or superior to conventional ultrasound assisted extraction. We developed an SFE protocol suitable for all metabolites that resulted in higher extraction yields for most target compounds. The choice of co-solvent herein plays an important role. For more polar compounds such as the cittilins or DKxanthenes, $\mathrm{MeOH}$ is the best co-solvent for extraction amongst the co-solvents tested in our study. Here we also observe that the use of $50 \% \mathrm{MeOH}$ is superior to $20 \% \mathrm{MeOH}$. For myxobacterial natural products with a logP higher than 3, namely the myxovirescins, myxalamides, indiacens and myxochromids, the most efficient co-solvent for extraction is EtOAc. Estimating the polarity of the secondary metabolite to be extracted therefore is crucial for the choice of a matching co-solvent. SFE offers the possibility to extract secondary metabolites from bacteria while simultaneously introducing some degree of selectivity, whereas solvent extractions have a much broader extraction scope. Non-targeted analysis of the extracts underpin the notion that the choice of co-solvent (or rather the use of co-solvent at all) plays the most important role in SFE. Only less than one percent of myxobacterial compounds are extractable with pure $\mathrm{CO}_{2}$ alone. Besides the many compounds extractable under all conditions, most myxobacterial secondary metabolites in this study are extractable when adding a polar co-solvent like ${ }^{i} \mathrm{PrOH}$ or $\mathrm{MeOH}$. In comparison to UAE we find $12 \%$ more features uniquely extractable with SFE, highlighting that SFE should be preferred over UAE in order to access as many metabolites as possible. We also observe an increase in antimicrobial activities of the SF extracts compared to the UA extracts. Importantly, some antimicrobial activities could only be seen in the SF extracts indicating that certain bioactive metabolites can only be detected and isolated using the SF-based approach. This is exemplified by strain MSr11367, where we could not observe any bioactivities from the UAE at all.

Our non-targeted analysis of the extracts shows that the overall number of metabolites that are exclusively extractable with SFE is comparably low. Nevertheless, this result highlights that the most powerful feature of SFE is its capability to increase the metabolite concentration in the resulting extract fractions thereby making new metabolites accessible. This finding is also reflected in the increased bioactivities seen in SF extracts. Many secondary metabolites are generally detectable with highly sensitive mass spectrometry but may not give rise to bioactivity in assays as these often suffer from comparably low sensitivity, especially in case of cell based assays aiming to identify antifungals and antibacterials. The use of SFE can therefore help to detect and prioritize new natural products based on their antimicrobial activities. Furthermore, our study shows that SFE can be used to prioritize secondary metabolites for isolation and structure elucidation based on their chemical properties, as demonstrated here for chloroxanthic acid A. In this case, SFE achieved concentrations in the extract at which isolation and structure elucidation became feasible, without the necessity to upscale to very high culture volumes. Especially for myxobacteria - where the change in cultivation conditions during upscaling often results in a decrease of secondary metabolite production - SFE thus paves the way to access secondary metabolites that have not been uncovered 
to date. ${ }^{44}$ Taken together, the advantageous features of SFE encourage its use for a wider range of microbial natural products applications in the future.

\section{References}

(1) Rafińska, K.; Pomastowski, P.; Rudnicka, J.; Krakowska, A.; Maruśka, A.; Narkute, M.; Buszewski, B. Effect of solvent and extraction technique on composition and biological activity of Lepidium sativum extracts. Food Chem. 2019, 289, 16-25.

(2) Manjare, S. D.; Dhingra, K. Supercritical fluids in separation and purification: A review. Materials Science for Energy Technologies 2019, 2, 463-484.

(3) Khaw, K.-Y.; Parat, M.-O.; Shaw, P. N.; Falconer, J. R. Solvent Supercritical Fluid Technologies to Extract Bioactive Compounds from Natural Sources: A Review. Molecules (Basel, Switzerland) 2017, 22.

(4) Chollet, C.; Boutet-Mercey, S.; Laboureur, L.; Rincon, C.; Méjean, M.; Jouhet, J.; Fenaille, F.; Colsch, B.; Touboul, D. Supercritical fluid chromatography coupled to mass spectrometry for lipidomics. J. Mass Spectrom. 2019, 54, 791-801.

(5) Wrona, O.; Rafińska, K.; Walczak-Skierska, J.; Możeński, C.; Buszewski, B. Extraction and Determination of Polar Bioactive Compounds from Alfalfa (Medicago sativa L.) Using Supercritical Techniques. Molecules (Basel, Switzerland) 2019, 24.

(6) Dutta, S.; Bhattacharjee, P. Enzyme-assisted supercritical carbon dioxide extraction of black pepper oleoresin for enhanced yield of piperine-rich extract. J Biosci Bioeng 2015, 120, 17-23.

(7) Yang, X.; Li, Y.; Li, Y.; Ye, D.; Yuan, L.; Sun, Y.; Han, D.; Hu, Q. Solid Matrix-Supported Supercritical $\mathrm{CO}_{2}$ Enhances Extraction of $\gamma$-Linolenic Acid from the Cyanobacterium Arthrospira (Spirulina) platensis and Bioactivity Evaluation of the Molecule in Zebrafish. Marine drugs 2019, 17.

(8) Cocks, S.; Wrigley, S. K.; Chicarelli-Robinson, M. I.; Smith, R. M. High-performance liquid chromatograpy comparison of supercritical-fluid extraction and solvent extraction of microbial fermentation products. J. Chromatogr. A 1995, 697, 115-122.

(9) Knudsen, P. B.; Hanna, B.; Ohl, S.; Sellner, L.; Zenz, T.; Döhner, H.; Stilgenbauer, S.; Larsen, T. O.; Lichter, P.; Seiffert, M. Chaetoglobosin A preferentially induces apoptosis in chronic lymphocytic leukemia cells by targeting the cytoskeleton. Leukemia 2014, 28, 1289-1298.

(10) Li, B.; Gao, Y.; Rankin, G. O.; Rojanasakul, Y.; Cutler, S. J.; Tu, Y.; Chen, Y. C. Chaetoglobosin K induces apoptosis and $\mathrm{G} 2$ cell cycle arrest through p53-dependent pathway in cisplatin-resistant ovarian cancer cells. Cancer Lett. 2015, 356, 418-433.

(11) Silva, F. V. M.; Martins, A.; Salta, J.; Neng, N. R.; Nogueira, J. M. F.; Mira, D.; Gaspar, N.; Justino, J.; Grosso, C.; Urieta, J. S.; et al. Phytochemical profile and anticholinesterase and antimicrobial activities of supercritical versus conventional extracts of Satureja montana. J. Agric. Food Chem. 2009, 57, 11557-11563. 
(12) Tadić, V.; Bojović, D.; Arsić, I.; Dorđević, S.; Aksentijevic, K.; Stamenić, M.; Janković, S. Chemical and antimicrobial evaluation of supercritical and conventional Sideritis scardica Griseb., Lamiaceae extracts. Molecules (Basel, Switzerland) 2012, 17, 2683-2703.

(13) Wrona, O.; Rafińska, K.; Możeński, C.; Buszewski, B. Supercritical Fluid Extraction of Bioactive Compounds from Plant Materials. J. AOAC Int. 2017, 100, 1624-1635.

(14) Esquivel-Hernández, D. A.; Rodríguez-Rodríguez, J.; Cuéllar-Bermúdez, S. P.; García-Pérez, J. S.; Mancera-Andrade, E. I.; Núñez-Echevarría, J. E.; Ontiveros-Valencia, A.; Rostro-Alanis, M.; García-García, R. M.; Torres, J. A.; et al. Effect of Supercritical Carbon Dioxide Extraction Parameters on the Biological Activities and Metabolites Present in Extracts from Arthrospira platensis. Marine drugs 2017, 15.

(15) Aresta, M. Carbon dioxide as chemical feedstock; Green chemistry; Wiley-VCH: Weinheim, 2010.

(16) Pourmortazavi, S. M.; Hajimirsadeghi, S. S. Supercritical fluid extraction in plant essential and volatile oil analysis. J. Chromatogr. A 2007, 1163, 2-24.

(17) Alvarez-Sanchez, B.; Priego-Capote, F.; de Castro,M. D. L. Metabolomics analysis II. Preparation of biological samples prior to detection. Trac-Trends Anal Chem 2010, 29, 120-127.

(18) Tatsuya Ito; Miyako Masubuchi. Dereplication of microbial extracts and related analytical technologies. J Antibiot 2014, 67, 353-360.

(19) Seidel, V. Initial and Bulk Extraction of Natural Products Isolation. In Natural products isolation, 3rd ed. / edited by Satyajit D. Sarker, Lutfun Nahar; Sarker, S. D., Nahar, L., Eds.; Methods in molecular biology, 1064-3745 864; Humana Press: New York, 2012; pp 27-41.

(20) Zhang, L. Integrated Approaches for Discovering Novel Drugs From Microbial Natural Products. In Natural Products; Zhang, L., Demain, A. L., Eds.; Humana Press Inc: [New York], 2005; pp 33-55.

(21) Panter, F.; Krug, D.; Müller, R. Novel Methoxymethacrylate Natural Products Uncovered by Statistics-Based Mining of the Myxococcus fulvus Secondary Metabolome. ACS Chem. Biol. 2019, 14, 88-98.

(22) Zhang, F.; Braun, D. R.; Rajski, S. R.; DeMaria, D.; Bugni, T. S. Enhypyrazinones A and B, Pyrazinone Natural Products from a Marine-Derived Myxobacterium Enhygromyxa sp. Marine drugs 2019, 17.

(23) Surup, F.; Chauhan, D.; Niggemann, J.; Bartok, E.; Herrmann, J.; Koeck, M.; Zander, W.; Stadler, M.; Hornung, V.; Müller, R. Activation of the NLRP3 inflammasome by hyaboron, a new asymmetric boron-containing macrodiolide from the Myxobacterium Hyalangium minutum. ACS Chem. Biol. 2018, 13, 2981-2988.

(24) Gorges, J.; Panter, F.; Kjaerulff, L.; Hoffmann, T.; Kazmaier, U.; Müller, R. Structure, Total Synthesis, and Biosynthesis of Chloromyxamides: Myxobacterial Tetrapeptides Featuring an Uncommon 6Chloromethyl-5-methoxypipecolic Acid Building Block. Angew. Chem. Int. Ed. Engl. 2018, 57, 1427014275. 
(25) Kjaerulff, L.; Raju, R.; Panter, F.; Scheid, U.; Garcia, R.; Herrmann, J.; Müller, R. Pyxipyrrolones: Structure elucidation and biosynthesis of cytotoxic myxobacterial metabolites. Angew. Chem. Int. Ed. 2017, 56, 9614-9618.

(26) Nadmid, S.; Plaza, A.; Garcia, R.; Müller, R. Cystochromones, unusual chromone-containing polyketides from the myxobacterium Cystobacter sp. MCy9104. J. Nat. Prod. 2015, 78, 2023-2028.

(27) Bader, C. D.; Panter, F.; Müller, R. In depth natural product discovery - Myxobacterial strains that provided multiple secondary metabolites. Biotechnol. Adv. 2020, 39, 107480.

(28) Wenzel, S. C.; Müller, R. Myxobacteria - "microbial factories' for the production of bioactive secondary metabolites. Mol. Biosyst. 2009, 5, 567-574.

(29) Abbas, C. A.; Sibirny, A. A. Genetic control of biosynthesis and transport of riboflavin and flavin nucleotides and construction of robust biotechnological producers. Microbiol Mol Biol Rev 2011, 75, 321-360.

(30) Krug, D.; Zurek, G.; Revermann, O.; Vos, M.; Velicer, G. J.; Müller, R. Discovering the Hidden Secondary Metabolome of Myxococcus xanthus: a Study of Intraspecific Diversity. Appl. Environ. Microbiol. 2008, 74, 3058-3068.

(31) Hug, J. J.; Dastbaz, J.; Adam, S.; Revermann, O.; Koehnke, J.; Krug, D.; Müller, R. Biosynthesis of cittilins, unusual ribosomally synthesized and post-translationally modified peptides from Myxococcus xanthus. ACS Chem. Biol. 2020, accepted.

(32) Trowitzsch, W.; Wray, V.; Gerth, K.; Höfle, G. Structure of myxovirescin A, a new macrocylic antibiotic from gliding bacteria. J. Chem. Soc., Chem. Commun. 1982, 1340.

(33) Meiser, P.; Bode, H. B.; Müller, R. The unique DKxanthene secondary metabolite family from the myxobacterium Myxococcus xanthus is required for developmental sporulation. Proc. Natl. Acad. Sci. U.S.A. 2006, 103, 19128-19133.

(34) Jansen, R.; Reifenstahl, G.; Gerth, K.; Reichenberg, H.; Höfle, G. Myxalamide A,B,C und D, eine Gruppe homologer Antibiotika aus Myxococcus xanthus Mx x12 (Myxobacterales). Liebigs Ann. Chem. 1983, 1081-1095.

(35) Kim, J.; Choi, J. N.; Kim, P.; Sok, D. E.; Nam, S. W.; Lee, C. H. LC-MS/MS Profiling-Based Secondary Metabolite Screening of Myxococcus xanthus. J. Microbiol. Biotechnol. 2009, 19, 51-54.

(36) Trowitzsch Kienast, W.; Gerth, K.; Reichenbach, H.; Höfle, G. Myxochromid A: Ein hochungesättigtes Lipopeptidlacton aus Myxococcus virescens. Liebigs Ann. Chem. 1993, 1233-1237.

(37) Iwasa, K.; Takahashi, T.; Nishiyama, Y.; Moriyasu, M.; Sugiura, M.; Takeuchi, A.; Tode, C.; Tokuda, $\mathrm{H}$.; Takeda, K. Online structural elucidation of alkaloids and other constituents in crude extracts and cultured cells of Nandina domestica by combination of LC-MS/MS, LC-NMR, and LC-CD analyses. J. Nat. Prod. 2008, 71, 1376-1385. 
(38) Steinmetz, H.; Mohr, K. I.; Zander, W.; Jansen, R.; Gerth, K.; Müller, R. Indiacens A and B: prenyl indoles from the myxobacterium Sandaracinus amylolyticus. J. Nat. Prod. 2012, 75, 1803-1805.

(39) RAMSEY, E. D. Analytical Supercritical Fluid Extraction Techniques; Springer Netherlands: Dordrecht, 2012.

(40) Helka Turunen. CO2-Balance in the Atmosphere and CO2-Utilisation: an engineering approach. Academic dissertation, University of Oulu, Linnanmaa, 2011.

(41) Gerth, K.; Jansen, R.; Reifenstahl, G.; Höfle, G.; Irschik, H.; Kunze, B.; Reichenbach, H.; Thierbach, G. The myxalamids, new antibiotics from Myxococcus xanthus (Myxobacterales). I. Production, physico-chemical and biological properties, and mechanism of action. J. Antibiot. 1983, 36, 1150-1156.

(42) Xiao, Y.; Gerth, K.; Müller, R.; Wall, D. Myxobacterium-produced antibiotic TA (myxovirescin) inhibits type II signal peptidase. Antimicrob. Agents Chemother. 2012, 56, 2014-2021.

(43) Meiser, P.; Weissman, K. J.; Bode, H. B.; Krug, D.; Dickschat, J. S.; Sandmann, A.; Müller, R. DKxanthene biosynthesis-understanding the basis for diversity-oriented synthesis in myxobacterial secondary metabolism. Chem. Biol. 2008, 15, 771-781.

(44) Hug, J. J.; Bader, C. D.; Remškar, M.; Cirnski, K.; Müller, R. Concepts and Methods to Access Novel Antibiotics from Actinomycetes. Antibiotics 2018, 7, 44. 